\title{
Mobility Management Solutions in Industrial Wireless Sensor Networks
}

\author{
Zinon Zinonos \\ Department of Information Sciences \\ Neapolis University Pafos \\ Pafos, Cyprus \\ zinonas@nup.ac.cy
}

\author{
Vasos Vassiliou \\ Department of Computer Science \\ University of Cyprus \\ Nicosia, Cyprus \\ vasosv@cs.ucy.ac.cy
}

\begin{abstract}
A number of sensor network applications are envisioned to be applied to industry settings where the existence of mobile nodes (MN) is required. In critical applications, the realtime monitoring of a MN must always be available, something that requires the existence of a suitable mobility protocol to control the handoff procedure. In this paper, we use an industrial WSN setting to perform a comprehensive performance evaluation of different mobility handling solutions based on single- and multiple-metric options. The results show that Fuzzy Logic-based Mobility Controller (FLMC), the multiple-metric approach we used (based on Fuzzy Logic), performs better compared to any single metric-based approach under a varying set of conditions. More specifically, we demonstrate that the Fuzzy Logic -based approach can efficiently control the handoff triggering procedure and provide high reliability (low packet loss) under different mobility models, different radio propagation models, and different topologies.
\end{abstract}

Index Terms-Sensor Networks, Mobility Management, Fuzzy Logic

\section{INTRODUCTION}

Wireless sensor networks offer an appropriate way to monitor physical objects and environments. Applications of wireless sensor networks have progressed in different domains due to their large applicability and development possibilities. In the majority of the applications, static sensor arrays are deployed to collect sensor readings from large or remote geographical areas to a central point. Recently, the WSN applications have experienced a paradigm change from static deployments to dynamic environments, meaning that mobile sensor nodes exist.

By introducing mobility to WSNs, we can further improve the network capability on many aspects of network performance. In order to achieve that, an appropriate mobility protocol is required. The aim of mobility support protocols is to keep nodes reachable and connected during the handover process, without interruption of connectivity.

Mobility management deals with all actions that must be taken in a network to support the movement of mobile users without losing connectivity. When a mobile user/node moves to a new location it has to establish a new radio link with the target base-station/access-point/neighbor and release the connection with the previous, in a process called handoff. A basic handoff process consists of three main phases: (a) triggering phase, dealing with initiating the handoff, (b) the decision phase, dealing with the algorithm parameters and handover criteria, and (c) execution phase dealing with the executions of the handoff [1].

Mobility support in an industrial environment is not of the upmost importance of the existing industrial standards like WirelessHart [2] and ISA100 [3]. WirelessHART and ISA100.11a use a centralized network management approach for communication scheduling. Despite the advantages of such approach when the network topology and application requirements are static and heavily pre-configured, it is not certain how these standards perform in dynamic situations involving node mobility. The failure to properly handle mobility results performance problems like increased packet loss, delayed data delivery, and increased downtime, all of which increase the overall energy consumption.

In this paper, we perform an extensive performance evaluation and comparison of different mobility solutions based on single- and multiple-metric options. Our aim is to compare the different handoff algorithms and extract conclusions regarding their performance under different situations. More specifically, we want to demonstrate via enriched simulative evaluation that the Fuzzy Logic -based approach can efficiently control the handoff triggering procedure and provide high reliability under different mobility models, different radio propagation models and different topologies.

The paper is organized as follows. In Section II, the related work is presented. In Section III, background information is presented where in Section IV the basic methods for handoff control in industrial WSNs are discussed along with the fuzzy logic-based mobility approach. In Section V, the experimental evaluation and performance analysis are presented. Finally, in Section VI the conclusions of this work are offered.

\section{RELATED WORK}

Several works using fuzzy logic techniques appeared in the field of mobility management, with the majority targeting the support of vertical handoffs. In [4], a handoff decision for heterogeneous networks is identified as a fuzzy multiple attribute decision-making problem and fuzzy logic is applied to deal with the imprecise information. In [5], a handover algorithm is proposed to support vertical handovers between heterogeneous networks. This is achieved by incorporating the 
mobile IP principles in combination with fuzzy logic concepts utilizing different handover parameters. Furthermore, in [6], the authors deal with a vertical handover decision algorithm based on the fuzzy control theory. The algorithm takes into consideration the factors of power level, cost, and bandwidth in order to decide about the vertical handover. In [7] the authors present a fuzzy logic system to support the mobility procedure based on several parameters like the RSSI level, the velocity of the mobile node, the number of hops to the sink node, and some other metrics such as traffic load, energy level, and link quality value.

The problem is that the applicability of the solutions cannot be investigate because there are not any practical implementation or evaluation of them. An additional issue is the fact that most of the solutions use high number of metrics that lead to an increased complexity of the fuzzy logic system, since a big number of rules must be enabled at any time. Due to the limited capabilities of the sensor nodes a fuzzy logic-based system must be as simple as possible.

In this work, we use a fuzzy-based solution that does not change the existing conventional algorithms, but uses operations of them in order to provide a system that will manage to control the handoff procedure and provide improved performance. In addition, our target is to provide a distributed solution, meaning that there should not be any central entity with full knowledge of the system that has to decide about the handoff procedure. Therefore all the information used is locally available at each node and no communication overhead is added.

\section{BACKGROUND INFORMATION}

\section{A. Network Topology}

The main architectural characteristics that were assumed in the system design are the following:

1) Use of a TDMA-based MAC protocol. Time is divided into epochs where each epoch has a predefined number of slots. Every node is assigned specific slots to transmit and receive packets. A number of slots is also assigned to each node (at the beginning of each epoch) for processing purposes.

2) The network uses multi-hop communication through a tree-based topology (Figure 1). The tree consists of $\mathrm{H}$ layers, where $\mathrm{H}$ is equal to the number of hops from the sink. A reasonable small number of nodes $(\mathrm{N}<30)$ is used where $\mathrm{N}$ is directly proportional to the required communications delay bound; the smaller the required delay, the smaller the $\mathrm{N}$.

3) The network topology is controlled dynamically. Each node is attached to the best available tree position during the construction of the network topology. Dynamic topology control is responsible for Neighbour Discovery, joining and leaving the tree, re-attachment (as in the case of mobility) to the tree, and maintenance of the topology in case of faults.
4) The network is made up of resource constrained embedded systems where the majority of the nodes are deployed in fixed and predetermined positions.

5) Nodes report data frequently with relatively high rate (up to once per second) and data must reach the sink within a given time bound Ts.

6) Each node can set its slots to the following modes: trasmitting, receiving, idle, and scanning. The scanning slots in The topology control and mobility modules are used to discover the neighbouring nodes and therefore to construct and maintain the tree topology. This is achieved by the fact that when the node sets its mode to scanning, it can receive any packet from any node inside its communication range.

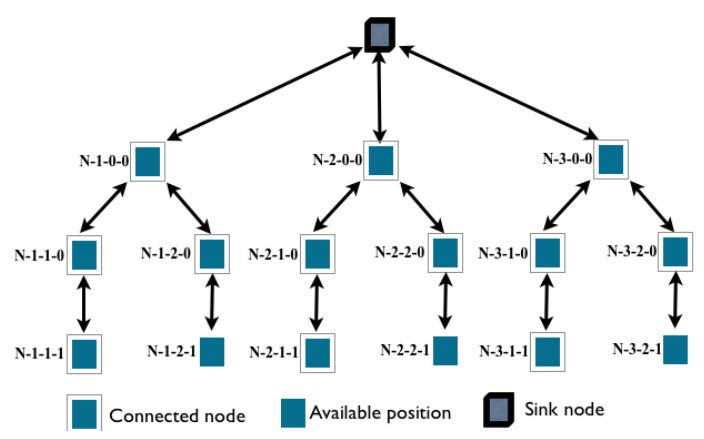

Figure 1: 3-2-1 Tree Topology

\section{B. Refinery Radio Propagation model}

In order to utilize the refinery radio propagation model, we proposed in [8] a new radio propagation model that was based on long-term refinery measurements in order to be integrated in COOJA simulator. The proposed radio model was validated by matching the performance of the simulated network to that of the real network, both in conditions involving static nodes and those in which mobile nodes were also present. We used statistical tests and exploratory statistical analysis, and we concluded that the refinery data are normally distributed. As such, the received signal strenght of the refinery radio propagation model is obtained by the following formula:

$$
R S S I(x)=\frac{1}{\sigma \sqrt{2 \pi}} e^{-(x-\mu)^{2} / 2 \sigma^{2}}
$$

\section{Mobility Solutions}

\section{A. Single Metric-based Solutions}

The first phase of a handoff deals with the triggering/initialization of the whole process. A range of metrics could potentially be used in the triggering procedure. Authors of [9], focused on two easy-to-find local values, namely the RSSI and the Local Link Loss (LL) in order to support the triggering of the handoff. Using these two metrics, they envisioned several triggering variations like their Simple Moving Average (SMA), Estimated Weighted Moving Average (EWMA) and Burst losses. 
In this work, we will use both RRSI-based and Link Lossbased solutions to compare them with the multi-metric based solution. More specifically, we will use the following options:

1) RSSI -78dBm: trigger the handoff when the RSSI value is less than $-78 \mathrm{dBm}$.

2) LL 1\%: trigger the handoff when the Link Loss is more than $1 \%$.

3) Simple Moving Average (SMA) RSSI with $n=10$ : trigger the handoff when the moving average of the $n$ latest values of the RSSI is less than $-78 \mathrm{dBm}$.

4) SMA Link Loss $10 \%$ with $n=10$ : trigger the handoff when the moving average of the $n$ latest values of the Link Loss is higher than $10 \%$.

\section{B. Fuzzy Logic-based Mobility Controller Solution (FLMC)}

Due to the highly dynamic nature of industrial environments the use of fuzzy logic to control the triggering procedure is an appropriate approach [10].

The Fuzzy Logic Controller provides the switching logic, which at any time chooses the best attachment point in the sense that it is the one that minimizes the Link Loss and increases the RSSI. The output of the fuzzy controller is a value that, compared with a predefined threshold, indicates whether the MN will initiate the triggering procedure. When the triggering starts the MN searches for a new attachment point. It decides to handoff if the new attachment point (if any) fulfills the handoff criteria.

The selection of fuzzy logic is supported by the fact that it can handle multiple inputs with minimum overhead. Thus, we can utilize a two-input, single-output fuzzy controller on each MN sensor in WSNs [10].

The FLMC is shown in Figure 2, where all quantities are considered at the discrete instant $\mathrm{kT}$ :

1) $T$ is the sampling period. The sampling period is equal to the time bound Ts.

2) $R S S I(k T)$ is the signal strength indication and $L L(k T)$ is the link loss rate.

3) $\operatorname{Pd}(k T)$ is the calculated decision point that triggers the handoff procedure. In the evaluation section, we will use $P d=0.16$ and $P d=0.18$ [10]

4) $S G i_{1,2}(k T)$ are the input scaling gains.

5) $P_{\text {Threshold }}$ is a predefined threshold that indicates if the the specific $\operatorname{Pd}(k T)$ will trigger the handoff

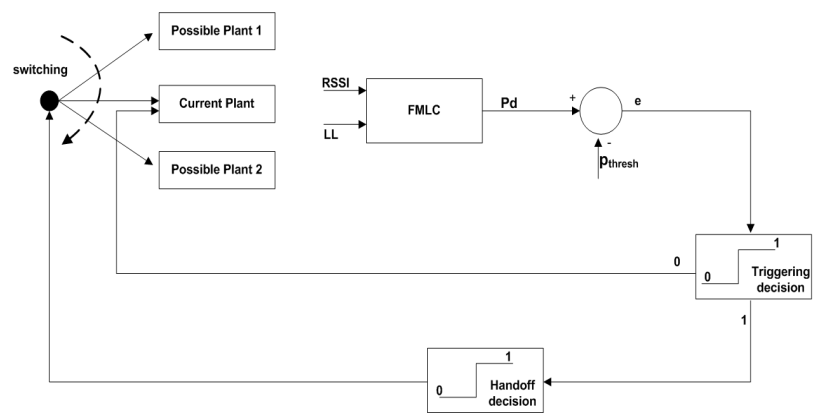

Figure 2: Fuzzy Logic-based Mobility controller (FLMC)
The FLMC follows a distributed approach that allows the system to adapt quickly to disturbances or changes within the network in real-time. This approach also includes some other critical targets like learning how the testbed environment operates.

We selected triangular and trapezoidal shaped membership functions in FLMC control system. The selected membership functions representing the linguistic values for both the inputs and the output of the FLMC controller are shown in Figure 3, Figure 4 and Figure 5.

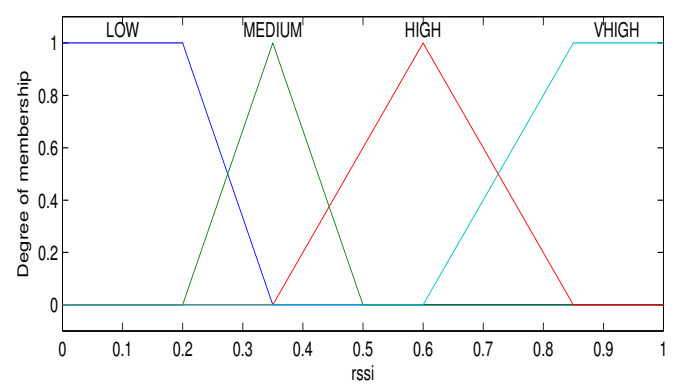

Figure 3: RSSI Linguistic Input

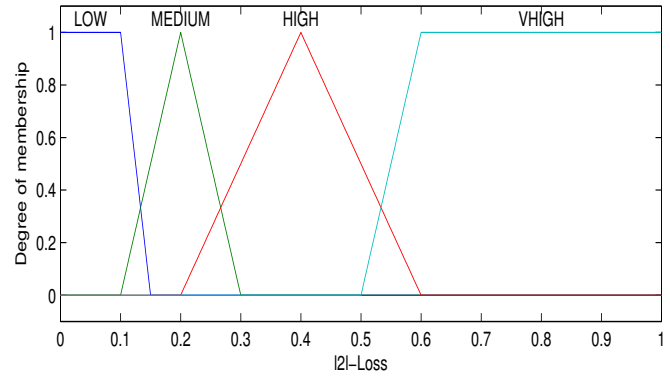

Figure 4: Link Loss Linguistic Input

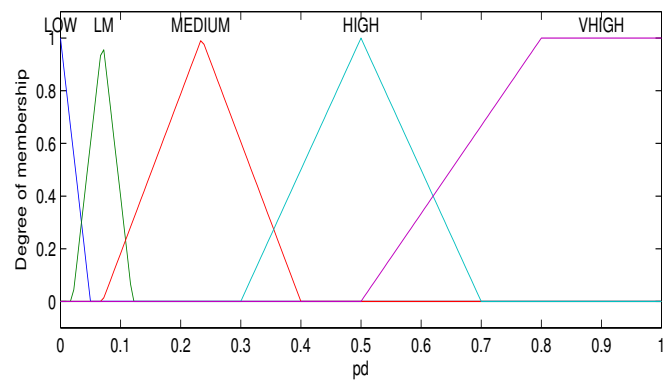

Figure 5: Decision Probability Linguistic Output

The list of all possible "IF-THEN" control rules are shown in Table I.

In order to design the FLMC, we followed specific steps that are briefly described below:

1) We used the industrial environment (specifically, an oil refinery) for over three months to collect data related 


\begin{tabular}{|c|c|c|c|c|c|}
\hline \multirow{2}{*}{\multicolumn{2}{|c|}{ Decision Probability }} & \multicolumn{4}{|c|}{ Link Loss Rate } \\
\hline & & $L^{a}$ & $M$ & $H$ & $V H$ \\
\hline \multirow{4}{*}{ RSSI } & $\bar{L}$ & LM & $\mathrm{M}$ & $\mathrm{H}$ & $\mathrm{VH}$ \\
\hline & $\bar{M}$ & LM & $\mathrm{M}$ & $\mathrm{H}$ & $\mathrm{VH}$ \\
\hline & $H$ & $\mathrm{~L}$ & $\mathrm{M}$ & $\mathrm{H}$ & $\mathrm{VH}$ \\
\hline & $V H$ & $\mathrm{~L}$ & LM & $\mathrm{H}$ & $\mathrm{VH}$ \\
\hline
\end{tabular}

Table I: FLMC Linguistic Rules - Rule Base

${ }^{a}$ low (L), low-medium (LM), medium (M), high (H), very high (VH)

to the RSSI, Link Loss and End-to-End loss. In this way, we were able to extra conclusions regarding their relationship and able to define the linguistic values and rules. After that, we quantified the meaning of the linguistic values and rules using membership functions.

2) We tuned the fuzzy controller in order to achieve an adequate performance based on the application requirements.

3) We investigated the stability of the FLMC controller in terms of phase plane analysis. We have proven that the states of the system remain within specific bounds [10].

It worths to mention that, there is no need for a fuzzy inference engine (FIE) to be built in each MN. By the time the linguistic rules and the linguistic values are defined, the control surface is known and can be stored as a lookup table (size of $n * n$, where $n=25$ in our case) requiring only a few kilobytes of memory in a fuzzy-capable MN. In that way, the memory and computation limitations of sensor networks are taken into account.

\section{Evaluation}

In the experiments, we used the COOJA [11] simulator and refinery data to mimic the behaviour of an industrial refinery setting. The basic parameters that were used for our simulations are shown in Table II. In order to run the simulation, we have imported into COOJA the following:

1) Initial node positions (Refinery placement)

2) Radio Propagation model [8]

3) FLMC implementation

Table II: Simulation Parameters

\begin{tabular}{|c|c|}
\hline Simulation Time & 2000 seconds \\
\hline Testbed Size & $35 \times 25$ meters \\
\hline Transmission Range & 20 meters \\
\hline Number of fixed/mobile nodes & $13 / 1$ \\
\hline Mobility model/Waypoint paths & Random Waypoint /10 \\
\hline Packet Rate & 1 packet / 3 seconds \\
\hline Topology & tree-based (3-2-1 tree) \\
\hline Number of free tree positions & 2 \\
\hline
\end{tabular}

\section{A. Evaluation using different Physical Topology}

The node placement in the refinery testbed was performed using a deterministic deployment, where sensors were precisely placed at pre-engineered positions capable of providing acceptable communication quality. When the network is consisted of a small number of nodes this deterministic approach is preferred, since one can easily determine whether the network is connected and, if not, to add relay nodes where needed. On the other hand, when having a large number of

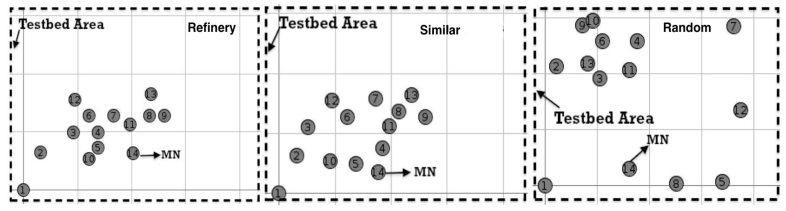

Figure 6: Topologies

nodes, it may be preferred to deploy the nodes randomly in order to reduce installation costs. Even though our network consisted of a small number of nodes, we decided to use also a randomly deployed topology in order to compare the mobility solutions.

Therefore, we repeated the experiments using two more new topologies. The first topology is a randomly constructed topology. For the second topology, we used the basic refinery topology with small changes in the placement of some nodes. Figure 6 shows the different topologies that were used. The purpose of this kind of evaluation is to observe whether the mobility solutions depend on the underlying topology or not and how they perform under different topologies. It should be mentioned that, since we have different placements/topologies, we can not compare the same solutions between them (ex. Fuzzy with $P_{\text {threshold }}=0.16$ using the basic topology with the Fuzzy with $P_{\text {threshold }}=0.16$ using the similar topology) because a different topology means different logical tree and therefore, different experiment. Hence, what we want to observe is if the different solutions behave the same under different topologies. For example, is the packet loss of Fuzzy Logic-based solution with $P_{\text {threshold }}=0.16$ less than the RSSI threshold solution in random topology as it happens in the refinery topology?

In the refinery placement, we observe that the nodes are placed in the center section of the testbed with small distances between them. In the refinery similar topology, the nodes are once again placed in the center section of the testbed but distributed to a bigger placement area. The reason of these small changes is to maintain the connectivity and avoid the creation of "black holes" in the communication of the sensor nodes. Finally, in the random topology, the nodes were randomly distributed within the testbed area.

Figure 7 shows the results regarding the End-to-End packet loss. As we observe, in random placement, the packet losses have increased for all cases. This is due to the fact that the random placement of few nodes creates uncovered areas, therefore in these areas the $\mathrm{MN}$ communication is disconnected. In addition, the refinery similar placement outperforms the refinery placement in terms of packets lost. This is due to the better distribution of the nodes in the center of the testbed.

Table III shows the total number of triggers and handoffs. Using the random topology, the triggers have increased in all cases. In addition, we observe that the handoffs have also increased in all the solutions with the single metric-based solutions to show the highest increment. This is mostly due to the higher trigger increment of the single-based solutions 


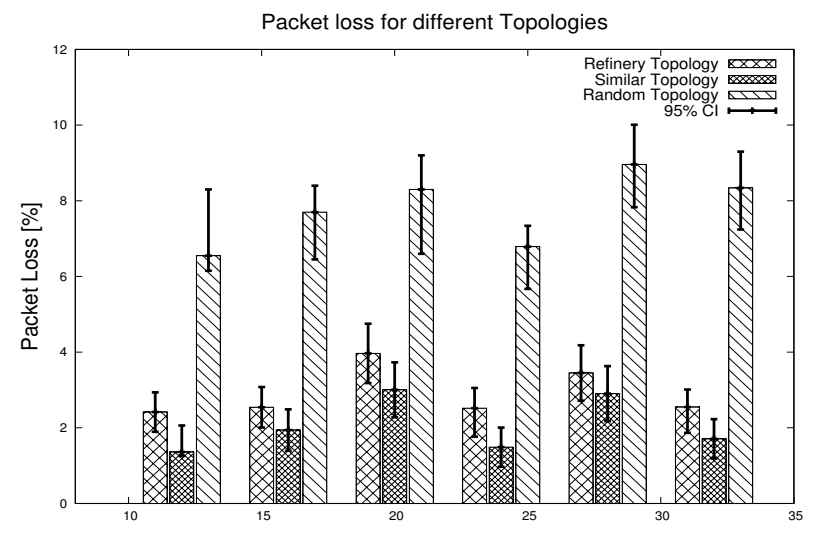

Figure 7: Packet Loss Comparison for Different Topologies

Table III: Number of Triggers/Handoffs Comparison for different Topologies

\begin{tabular}{|l|l|l|l|l|l|l|}
\hline & \multicolumn{2}{|c|}{ Refinery } & \multicolumn{2}{c|}{ Similar } & \multicolumn{2}{c|}{ Random } \\
\hline & Triggers & Handoffs & Triggers & Handoffs & Triggers & Handoffs \\
\hline FLMC, Pd=0.16 & 5.7 & 0.52 & 6.91 & 0.62 & 19.7 & 0.62 \\
\hline FLMC, Pd=0.18 & 2.3 & 0.41 & 3.05 & 0.34 & 16.8 & 0.53 \\
\hline RRSI, -78dBm & 9.85 & 0.15 & 12.87 & 0.21 & 32.34 & 0.42 \\
\hline LL=1\% & 2.25 & 0.36 & 7.39 & 0.52 & 15 & 0.79 \\
\hline SMA RSSI, $\mathrm{n}=10$ & 0.43 & 0.14 & 0.99 & 0.19 & 14.8 & 0.39 \\
\hline SMA LL=10\%, $\mathrm{n}=10$ & 22.17 & 0.41 & 20.57 & 0.57 & 26.2 & 0.59 \\
\hline
\end{tabular}

which lead to more scanning/receiving periods and, therefore, lead to a higher probability to handoff.

Finally, Figure 8 shows the total power consumption of the tests. We observe that when using the random placement, the power consumption is increased in all the solutions as a consequence of the increased number of triggers and the associated increased scanning/receiving period. In the case of the refinery similar topology, we observe a small improvement to the power consumption compared to the refinery topology. Furthermore, we observe that in case of the RSSI-based solutions the power consumption is less than any other solution. This is due to the fluctuations of the received signal which force the algorithm to perform lot of one epoch unnecessary triggers (see Table III). Therefore, with the RSSI-based solutions we have instant scanning periods which means low propability to find a better attachment point. This is the reason we see an increased packet loss in case of the RSSI-based solutions.

Comparing the overall performance of the different solutions using all the placements, we observe that the trends are the same. For example, in both placements, the FLMC solution outperforms the RSSI-based in terms of packet losses. The same happens when comparing the FLMC with the Link Loss option (4\%-9\% reduction of the packet loss). It is also obvious that the packet loss depends on the topology of the network.

\section{B. Comparison of different Radio models}

The FLMC was designed based on the radio propagation model of the refinery environment. In addition to that, in this section we use a simplified and different radio propagation model where the RSSI linearly depends on the distance between the transmitting and receiving node. This radio propagation model is the called Unit Disk Graph Medium

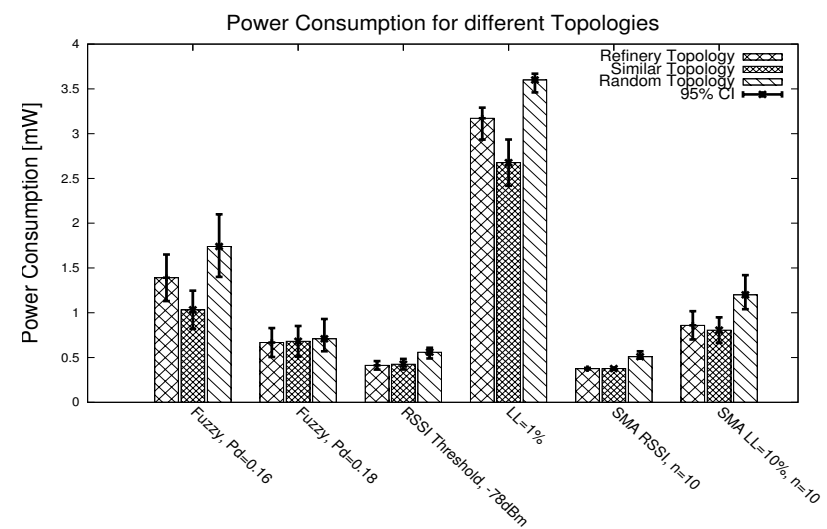

Figure 8: Power Consumption Comparison of Refinery vs Random Placement

(UDGM): Distance Loss. In our experiments, by using the distance based solution we set the transmission and reception success equal to 1 , which means that if two nodes are within the communication range of each other the packet will be delivered with a $100 \%$ success.

Figure 9 shows the packet losses comparison of the distancebased model and the refinery model. We observe that, the distance based provides fewer losses compared to the refinery model. The highest improvement is shown in the case of the RSSI Threshold solution. The reason for that is the absence of the RSSI fluctuations and the $100 \%$ delivery success, if the nodes are within communication range. Comparing the mobility solutions, we conclude that FLMC solutions perform better in terms of packets lost than any other solution.

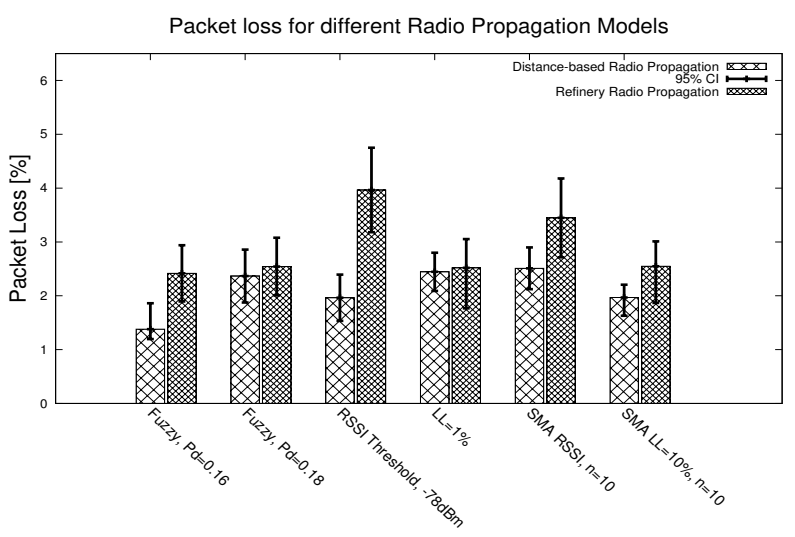

Figure 9: Packet Loss comparison of different Radio Propagation Models

Table IV shows the number of triggers and the number of handoffs for both radio propagation models. It is obvious that in the case of the FLMC solution, we have less triggers compared to the other solutions; that is, the FLMC controls better the handoff triggering procedure compared to the conventional solutions, by minimizing the number of unnecessary handoffs.

Finally, Figure 10 shows the power consumption comparison. 
Table IV: Number of Triggers/Handoffs Comparison for different Radio Propagation Models

\begin{tabular}{|l|l|l|l|l|}
\hline & \multicolumn{2}{|c|}{ Refinery } & \multicolumn{2}{c|}{ Distance-based } \\
\hline & Triggers & Handoffs & Triggers & Handoffs \\
\hline FLMC, $\mathrm{Pd}=0.16$ & 5.7 & 0.52 & 6.04 & 1.01 \\
\hline FLMC, $\mathrm{Pd}=0.18$ & 2.3 & 0.41 & 2.5 & 0.49 \\
\hline RRSI, -78dBm & 9.85 & 0.15 & 33.95 & 6.09 \\
\hline LL=1\% & 2.25 & 0.36 & 15.56 & 3.14 \\
\hline SMA RSSI, $\mathrm{n}=10$ & 0.43 & 0.14 & 30.30 & 4.83 \\
\hline SMA LL=10\%, $\mathrm{n}=10$ & 22.17 & 0.41 & 29.49 & 1.58 \\
\hline
\end{tabular}

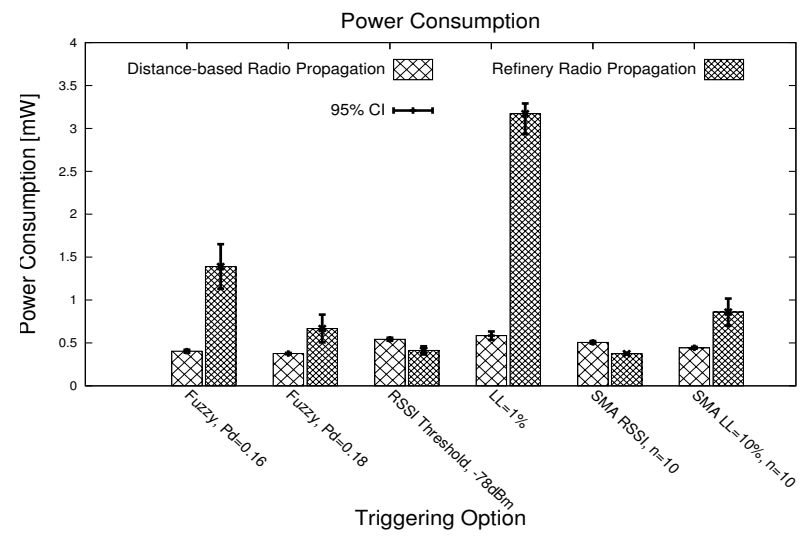

Figure 10: Power Consumption comparison of different Radio Propagation Models

We observe that, in FLMC solutions the power consumption is drastically reduced when using the distance-based radio propagation model. This is due to the smaller duration of the triggering periods since in distance-based model the input parameters of the FLMC (RSSI and Link Loss) change frequently compared to the refinery (Gaussian-based) model [8]. Therefore, the FLMC can exit from scanning mode easily. In addition, we observe that the power consumption of the RSSIbased solution is increased because of the increased number of triggers. Finally, the power consumption of the Link Loss triggering solutions is reduced mainly due to the different link loss formulation where, in case of the refinery, it is based on data obtained from the refinery and, in case of the distancebased it is based on the transmission range.

\section{Evaluation using different Mobility models}

In order to identify how the different mobility models affect the overall performance, we repeated the experiments using two different mobility models, the Gauss Markov and the Manhattan Grid models. For the Gauss Markov model, we set the maxSpeed $=3 \mathrm{~m} / \mathrm{s}$ and the speedStdDev $=0.5$. For the Manhattan Grid model, we set the grid size to $10 x 10$, the $\operatorname{minSpeed}=0.5 \mathrm{~m} / \mathrm{s}$, and the meanSpeed $=1.5$.

The results are shown in Figures 11-12 and in Table V.

It is obvious that Random Waypoint model shows better performance. This is due to the characteristic of Random Waypoint model to cluster the movement of the MN near the centre region of simulation field and move away from the boundaries (density wave phenomenon). Observing the nodes'

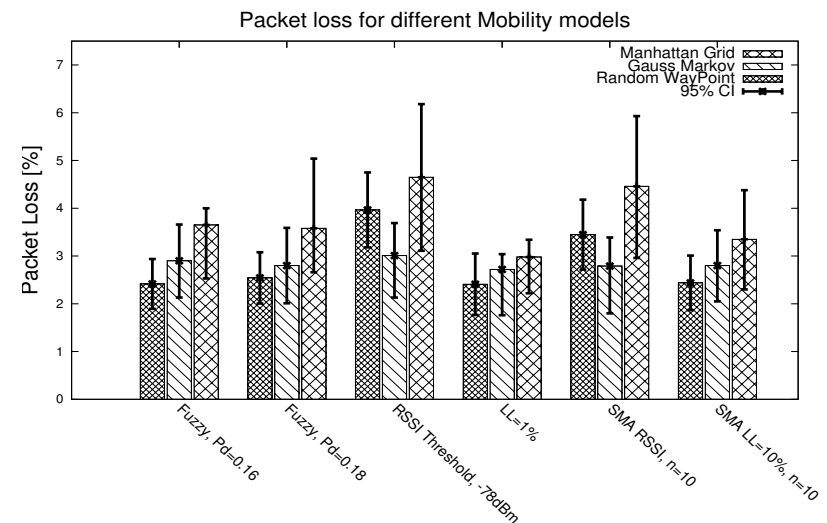

Figure 11: Packet Loss comparison of different Mobility Models

Table V: Number of Triggers/Handoffs Comparison for different Mobility Models

\begin{tabular}{|l|l|l|l|l|l|l|}
\hline & \multicolumn{2}{|c|}{ Manhattan Grid } & \multicolumn{2}{c|}{ Gauss Markov } & \multicolumn{2}{c|}{ Random Waypoint } \\
\hline & Triggers & Handoffs & Triggers & Handoffs & Triggers & Handoffs \\
\hline FLMC, Pd=0.16 & 6.6 & 0.45 & 16.58 & 0.64 & 5.7 & 0.52 \\
\hline FLMC, Pd=0.18 & 3.65 & 0.31 & 7.48 & 0.43 & 2.3 & 0.41 \\
\hline RRSI, -78dB & 10.03 & 0.24 & 35.8 & 0.49 & 9.85 & 0.15 \\
\hline LL=1\% & 13.8 & 0.8 & 22.3 & 0.926 & 2.25 & 0.36 \\
\hline SMA RSSI, $\mathrm{n}=10$ & 9.01 & 0.13 & 25.79 & 0.15 & 0.43 & 0.14 \\
\hline SMA LL=10\%, $\mathrm{n}=10$ & 35.5 & 0.7 & 33 & 0.45 & 22.17 & 0.41 \\
\hline
\end{tabular}

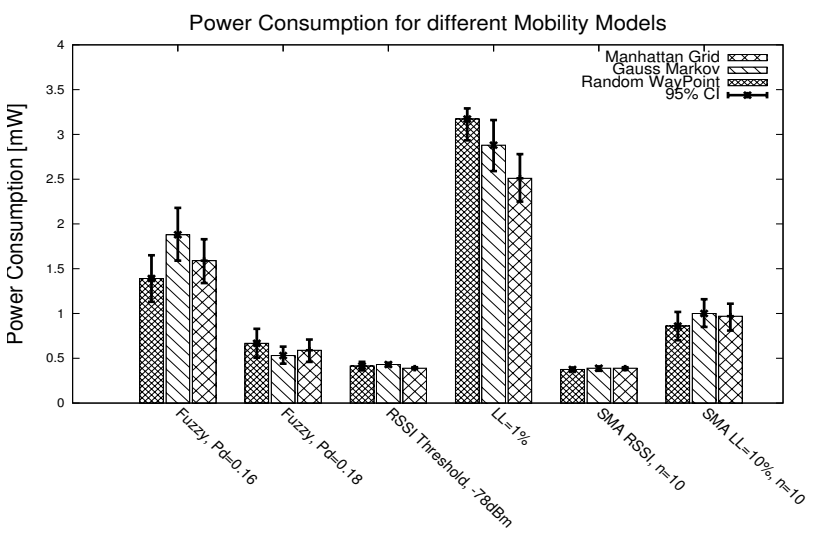

Figure 12: Power Consumption comparison of different Mobility Models

placement, we see that the center region of the testbed area has higher node density than the boundaries. Furthermore, based on [12] the Random Waypoint model provided the maximum connectivity (close to 90\%) among the nodes for low density networks compared to the Gauss-Markov (close to $70 \%$ ) and Manhattan Grid (close to 65\%) models. Thus, Random Waypoint model present lower packet losses due to connectivity issues compared to the other two mobility models.

\section{CONCLUSIONS}

In this paper, our objective has been to compare different mobility solutions so that to conclude which solution ensures low End-to-End packet losses. In all cases, the proposed Fuzzy Logic Mobility Control (FLMC) displayed the best 
performance. It is worth to mention that the FLMC approach can be used with any underlying communication technology, since it was designed to require only two general metrics, the RSSI and the Link Loss. Based on our experimentation, we can conclude that our two main evaluation parameters, the packet loss and the power consumption, depend on the network topology, on the handoff algorithm, and on the type of movement.

\section{REFERENCES}

[1] V. Vassiliou, J. Antoniou, A. Pitsillides, and G. Hadjipollas, "Simulating Soft Handover and Power Control for Enhanced UMTS," in Personal, Indoor and Mobile Radio Communications (PIMRC), 2005 IEEE 16th International Symposium on, Sept. 2005, pp. 1646-1651.

[2] J. Song, S. Han, A. Mok, D. Chen, M. Lucas, M. Nixon, and W. Pratt, "WirelessHART: Applying Wireless Technology in Real-Time Industrial Process Control," in Proceedings of the 2008 IEEE Real-Time and Embedded Technology and Applications Symposium, ser. RTAS '08, 2008, pp. 377-386.

[3] (2009) ISA-100.11a-2009: Wireless Systems for Industrial Automation: Process Control and Related Applications. [Online]. Available: http://www.isa.org/ISA100/

[4] W. Zhang, "Handover Decision Using Fuzzy MADM in Heterogeneous Networks," in Wireless Communications and Networking Conference, 2004. WCNC. 2004 IEEE, vol. 2, March 2004, pp. 653 - 658.

[5] P. M. L. Chan, R. Sheriff, Y. Hu, P. Conforto, and C. Tocci, "Mobility Management Incorporating Fuzzy Logic for Heterogeneous a IP Environment," Communications Magazine, IEEE, vol. 39 , no. 12, pp. 42-51, 2001.
[6] H. Liao, L. Tie, and Z. Du, "A Vertical Handover Decision Algorithm Based on Fuzzy Control Theory," in Computer and Computational Sciences, 2006. IMSCCS '06. First International Multi-Symposiums on, vol. 2, 2006, pp. 309-313.

[7] H. Fotouhi, M. Alves, A. Koubaa, and N. Baccour, "On a Reliable Handoff Procedure for Supporting Mobility in Wireless Sensor Networks," in the 9th International Workshop on Real-Time Networks RTN'2010 in conJunction with the 22nd Euromicro International Conference on Real-Time Systems (ECRTS 2010), Brussels, Belgium, 2010.

[8] Z. Zinonos, V. Vassiliou, and T. Christofides, "Radio Propagation in Industrial Wireless Sensor Network Environments: From Testbed to Simulation Evaluation," in Performance Monitoring and Measurement of Heterogeneous Wireless and Wired Networks (PM2HW2N), 2012 7th ACM workshop on, 2012, pp. 125-132.

[9] Z. Zinonos, V. Vassiliou, and C. Chrysostomou, "Handoff Triggering for Wireless Sensor Networks with Performance Needs," in 18th IEEE Symposium on Computers and Communications (IEEE ISCC 2013), Split, Croatia, Jul 2013.

[10] Z. Zinonos, C. Chrysostomou, and V. Vassiliou, "Wireless Sensor Networks Mobility Management Using Fuzzy Logic," Ad Hoc Networks, vol. 16, pp. 70-87, 2014.

[11] J. Eriksson, F. Österlind, N. Finne, N. Tsiftes, A. Dunkels, T. Voigt, R. Sauter, and P. J. Marrón, "COOJA/MSPSim: Interoperability Testing for Wireless Sensor Networks," in Proceedings of the 2nd International Conference on Simulation Tools and Techniques, ser. Simutools '09, 2009, pp. 27:1-27:7.

[12] N. Meghanathan, "Impact of the Gauss-Markov Mobility Model on Network Connectivity, Lifetime and Hop Count of Routes for Mobile Ad hoc Networks," Journal of Networks, vol. 5, no. 5, 2010. 